\title{
LES LÉSIONS NERVEUSES PERIPHERIQUES RELIÉES A L'ANESTHÉSIE*
}

\author{
BeVERLEY A BRITT, MD, D A (TOR), ET \\ R A GoRDon, BSC, MD, FRCP (C), I F A RCS
}

Très PEU de temps après le début de l'emplo1 de l'éther, ${ }^{1}$ on a observé des paralysies de nerfs à la suite de l'anesthésie Les rapporteurs de l'époque ont attribué ces lésions aux propriétés toxiques des agents employés

En 1894, Budınger ${ }^{2}$ a été le premier à reconnaître que la plupart de ces troubles nerveux postopératorres étaient dus à une mauvaise position du malade sur la table d'opération entraînant soit une élongation sort une compression des nerfs Ses découvertes furent aussitôt vérifiées par Krum, ${ }^{3}$ Garrıques, ${ }^{4}$ et Horsley ${ }^{5}$

Clausen, ${ }^{6}$ en 1942, et Ewing, ${ }^{7}$ en 1952, ont insisté sur l'umportance de l'élongation dans les lésions nerveuses au cours de l'anesthésie

D'autres travaux sur ce sujet ont été publiés jusqu’à nos jours mais nous observons encore de semblables blessures évitables

\section{LA Circulation DES NERFs}

Les nerfs reçoıvent des vaisseaux nourriciers à des intervalles irréguliers ma1s fréquents, ces vaisseaux sont spécialement abondants auprès des artıculations $\mathrm{Au}$ moment où ıls entrent dans un nerf, les vaisseaux sont de la talle d'une artériole Ces vaısseaux se divisent immédıatement en artérioles légèrement plus petıtes qui montent ou descendent longitudinalement dans les espaces interfasciculaures, et ils deviennent graduellement du type précapillaure Ils sanastomosent avec des vaisseaux parallèles et avec des artérioles plus distales et plus proxumales De ces artérioles, des branches capillaires plus petites passent directement dans le septum du tissu conjonctuf des faisceaux Ces branches capillaures ont également tendance à se disposer longitudinalement et, de plus, elles se divisent et s'anastomosent à plusieurs reprises Les anastomoses vascularres intraneurales, de fart, sont tellement abondantes que de longues portıons de nerfs peuvent être privées d'apport sanguin extra-neural et, en dépit de cela, demeurer viables ${ }^{13}$

\section{Pathogénie de la Blessure Nerveuse}

De l'élongatıon ou de la compression d'un nerf, ll résulte une sschémie Si cette 1schémie se prolonge assez longtemps, il se produit une nécrose du nerf

De plus, l'élongation d'un nerf entraîne des ruptures des vaisseaux capullaires et, subséquemment, il se forme un hématome dans le farsceau nerveux $\mathrm{S}_{1}$ Thématome est petat, les fibres nerveuses environnantes sont tout simplement

- Pour les figures ot la Bıblographre vorr l'artucle précédent. 
comprimées, et, plus tard, elles peuvent récupérer Toutefors, si l'hématome est considérable, les fibres nerveuses voisines deviennent complètement nécrosées Finalement, ll peut se produre un exsudat et de la cicatrisation des septa fibreux du tronc nerveux

Nous réalısons annsı que l'ischémı est plutôt d'orngine intra-neurale qu'extraneurale

\section{ETIOLOфIE}

\section{Anesthésie générale}

Le facteur princıpal quı produit la majorité des lésıons nerveuses périphériques chez les malades sous anesthésie est l'ischémie des vasa nervorum intraneuraux ${ }^{14,15}$ Cette ischémie est produite primitivement par l'élongation du nerf et secondaurement par la compression d'un nerf rendu vulnérable par l'élongation

L'élongation et la compression des nerfs sont plus exposées à survenir chez le malade anesthésié pour deux rasons ${ }^{\dagger}$ En premier heu, le tonus musculare est dımınué et le malade est plus sensible aux positions non physiologiques ${ }^{6} \mathrm{En}$ deuxıème lieu, pusque sa perception est troublée, le malade ne peut pas se plandre de positions douloureuses qu'il ne tolèrerait pas en temps ordinaure ${ }^{1}$

Même chez le malade conscient, l'abduction du bras à plus de $90^{\circ}$ en Trendelenburg marqué devient douloureux et intolérable au bout de quelques instants ${ }^{11}$ et le pouls radial disparaît chez 83 pour cent de ces cas Il ne faut pas plus de 30 ou 40 minutes d'anesthésie, chez un malade en mauvaise position, pour produrre une paralysie nerveuse ${ }^{11}$

\section{Anesthésıe rachıdıenne}

L'hypotension du liquide céphalo-rachıdien qui peut provenir d'une furte dans la dure-mère est probablement la cause des paralysıes des nerfs crânıens qui surviennent après les rachianesthésies On présume que cette hypotension produrt une descente de la moelle et du bulbe, ce qui cause une élongation des nerfs crâniens ${ }^{17-19}$

\section{Les injections locales}

Les paralysies nerveuses peuvent être causées par l'injection de substances dans un nerf ou autour d'un nerf La blessure peut être due à l'irritation chımique de la substance injectée, comme par exemple, un agent anesthésıque local, du thiopentone, de la noradrénaline, de la chlorpromazıne et du chlorure de $d$-tubocurarine Une argulle peut blesser directement un nerf Les nerfs 1adial et sciatıque peuvent être blessés par des injectıons intramusculaires faites au mauvais endroit Une infection au lieu de l'injection peut aussi devenir un facteur causal Enfin un hématome localısé, formé à la suite de la perforation d'une veine, peut comprimer un nerf au point de le rendre ischémıque ${ }^{2021}$

\section{L'hypothermie}

A la suite de l'hypothermie, on peut observer des neuropathies ${ }^{22}$ périphériques 
parce que, à cause des techniques actuelles de refroidıssement en surface, on observe des températures beaucoup plus basses en pé iphérie que dans le rectum ou l'oesophage, par exemple, $4^{\circ} \mathrm{C}$ dans le gastrocmiens contre $28^{\circ} \mathrm{C}$ dans le rectum, dans un cas particulıer ${ }^{23}$ L'addition de la plus légère pression, même celle de la courrose de caoutchouc qui retient l'électiode en place pour l'E K.G prédıspose probablement aux lésions nerveuses ${ }^{24}$

\section{L'hypotension}

L'hypotension accidentelle ou contrôlée, en dimmiuant le débit sanguin aux nerfs, peut produrre une paralysie nerveuse ou l'aggiaver s1 elle exıste déjà à cause de facteurs locaux

\section{Les tourniquets}

A l'occasion, nous entendons dire qu'un tourniquet a pu endommager les nerfs sur lesquels il était appliqué, sı la pression employée était excessive ou maintenue trop longtemps ${ }^{4142526}$ Toutefors, cecı est loin d'être aussı grave qu'une mauvase position, car le facteur élongation n'existe pas et la compression se repartit sur toute la curconférence du membre et s'exerce habituellement à travers une bonne couche de tissus mous et non pas directement contre un point dur osseux

\section{La toxucité}

Des lésıons nerveuses peuvent être produites par l'actıon toxıque directe des produits de dégradation des agents anesthésiques ou par d'autres impuretés qu'ils peuvent contenir Ainsı, le chloroforme exposé à la chaleur produit du phosgène, cecı a dû se produre dans les premiers temps de l'anesthésie au chloroforme, alors qu'on travallant entouré de chandelles et de bec, de gaz

En présence de chaleur et d'un alcalin tel que la chaux sodée, le trichloréthylène se décompose et forme du dichloracétylène qui est extrêmement toxıque pour le système nerveux centrall et qui, à son tour, par la chaleur, se transforme en phosgène et en oxyde de carbone ${ }^{2 \tau-30}$

\section{AsPeCtS CLINIQues}

La lésıon nerveuse peut se manifester immédıatennent après le réveıl ou seulement après plusieurs jours

Le malade se plaint d'un certain nombre de sensations désagréables dans les régions innervées par les nerfs affectés hyperesthésie, paresthésie, sensation de froıd, douleur ou anesthésıe Souvent, il ressent de la sensıbilité à la région affectée ${ }^{6}$

Il apparait souvent de la parésie et même de la paralysie franche avec perte des réllexes dans les muscles innervés par les nerfs lécés

On aura une preuve de la perte de l'mnervation sympathique si l'on constate une diminution de la transudation, une diminution de l'activité pilomotrice, de la rougeur et une augmentation de la $\mathrm{T}^{\circ}$ cutanée

Si la guérison n'est pas précoce, il peut survenir une atrophie musculare, des 
contractures musculaires, de la raıdeur des artıculations, de la déminéralısation des os, de la diminution du tissu sous-cutané, la peau peut s'atrophier, devenir lusante et s'ulcérer, on pourra observer une crosssance exagérée des ongles qui prendront une forme striée ${ }^{31}$

Pour faure un diagnostic entre les simulateurs et ceux qui sont réellement paralysés, il est utile de recourr à la plaque photographique au nitrate d'argent $S_{1}$ l'on place un membre normal (par exemple une main) sur cette plaque, à la norrceur, et qu'on développe cette plaque, on vort l'image de la main, parce que le chlorure de la sueur réagit avec le nitrate d'argent pour former du chlorure d'argent insoluble Cette réaction ne se produit pas dans les régions énervées à cause de l'absence de sueur

On peut aussı appliquer sur la régıon à examiner une solution de chlorure de cobalt saturé dans l'alcool à 95 pour cent La partie qui transpire passera du bleu au rouge ${ }^{31}$

\section{Pronostic}

La guérison peut survenir en quelques jours, en quelques semaines ou en quelques mois, ou encore, 1 peut persister une faiblesse permanente, un épuisement et une perte de sensibilité

Habituellement les forces reviennent très iapıdement dans les gros muscles, et plus lentement dans les petits muscles employés pour faure des mouvements délicats C'est pourquor, de façon générale, les travallleurs habiles sont plus handicapés que les travalleurs manuels Le pronostıc est d'autant plus mauvass que la période passée en mauvaise position est plus longue ${ }^{32}$ L'usage des myorésolutufs, ou de l'hypothermie assombrissent également le pronostic On observe plus fréquemment des séquelles permanentes chez des malades souftrant de maladies nerveuses, d'anémie, d'hypovolémie d'artériosclérose ${ }^{4}$ ou de déséquilıbre électrolytique ${ }^{33}$

\section{NEUROPATHIES SPÉCIFIQUES}

\section{Le membre supérneur}

\section{A Le plexus brachal}

Etrologie De tous les groupes de nerfs, le plexus brachial est de lom le plus vulnérable au cours des mauvaises positions durant l'anesthésie, et cela pour deux rassons D'abord, le plexus a un trajet axillaure relativement long, mobile et superficiel entre deux points fixes au-dessus, les vertèbres et le fascia prévertébral et, au-dessous, le fascia axillare En second lieu, le plexus repose dans le vorsinage ımmédiat d'un certain nombre de structures osseuses qui se déplacent librement

L'élongation est la principale cause de lésıons au plexus brachial, ${ }^{5,34} 36$ la compression joue un rôle secondarre Elle agit simplement pour former un point d'appur ou un point de stabilsation que le nerf doit contourner L'élongation du plexus brachial est produste pas tout facteur qui augmente la distance entre les points de fixatıon supéneure et inférneure, par exemple 
1 L'extension dorsale et la flexion latérale de la tête du côté opposé aggrandit l'angle entre la tête et le sommet de l'épaule, ce qui płoduit une tension sur le plexus ${ }^{36,5,69}$ (fig 1) *

2 L'abduction, la rotation externe et l'extension dorsale du bras sur un support -surtout s'il s'agit d'un angle de plus de $60^{\circ}$ par rapport à la table d'opérationla chute du bras à côté de la table causent une élongation extrême du plexus, (fig 4)

3 La suspension du bras à un cerceau, lorsque le malade est en position latérale (fig 5 )

4 L'abduction extrême des bras au-dessus de la têle lorsque le malade est couché sur le dos

5 Certans rapporteurs ont établi que le fait de fixer le malade par les poignets pous l'empêcher de glisser d'une position exagérée de Trendelenburg augmente la tension sur le plexus Nous discuterons ce détal de façon plus complète prochamement

Le plexus brachial peut être comprimé contre une ou plusieurs protubérances osseuses Exemple

1 Le plexus peut être coincé entre la clavicule et lis première côte lorsqu'on utılise des épauhères pour éviter que le malade ne glisse lorsqu'll est dans la position de Trendelenburg, et qu'au lieu de les placer au-dessus de l'articulation acromio-claviculaure, on les installe trop près de la ligne médiane, dans ce cas, elles repoussent la clavicule en bas et en arrière dans l'espace rétro-claviculaure ${ }^{26,9}$ Dans cette éventualité, lé plexus doit dévier par un trajet en forme de $S$ et, ainsi, la distance entre les points supérıeur et inférieur de fixatıon devient accrue $\mathrm{Si}$, en plus, le bras du malade est en abduction sur un support à bras tel que décrit c1-dessus, l'élongation devient extrême (fig 2)

2 Le plexus peut être poussé vers le bas en étant allongé au-dessus de la tête de l'humérus Ceci survient également lorsque le malade est en position de Trendelenburg et que son bras, en abduction, est étendu sur un support Les épaulières, dans ce cas, sont placées trop latéralement, elles portent en réalité sur la tête de l'humérus, la repoussent en bas vers l'aisselle, entraîncłnt le plexus avec elle ${ }^{\mathbf{3 6 , 3 7}}$ (fig 3)

3 Le plexus peut être repoussé en arrière par le tendon du petit pectoral tet même par l'extrémité de l'apophyse caracoide Ceci peut survenir en deux curconstances

a) Dans le cas d'un malade plutôt obèse, subissant une cholécystectomie, chez qui on a élevé le support du fore, $\mathbb{l}$ arrive que la tête de l'humérus déborde le matelas de la table d'opération pour reposer sur un support à bras qui n'est pas matelassé Tout le moignon de l'épaule tombe en arrière et latéralement par rapport à la cage thoracique L'insertıon du tendon du petıt pectoral à l'apophyse caracoide amsı que le plexus brachial sont donc poussés dans la même direction ${ }^{10}$ (fig 4)

b) Le même mécanısme se reproduit sı le malade est couché sur le côté, le bras suspendu à un cerceau ${ }^{6}$ Dans cette dernière circonstance, l'élément gravité accentue lischémie intraneurale (fig 5) 
4 Clausen $^{6}$ croyart que la première côte étart le point d'appuı sur lequel le plexus frottart lorsqu'une traction vers le bas était placée sur le bras en adduction pour empêcher le glıssement, dans la position de Trendelenburg Ewıng ${ }^{7}$ a discuté cette opinion et, d’après son expérience à la suite de dissectron sur les cadavres, ll n'y a pas d'augmentation importante de la tension sur le plexus lors d'une telle position, et, la première côte ne comprime le plexus en aucune façon

Certaines anomalies congénitales peuvent rendre le plexus plus vulnérable Ces anomalies sont

1 L'hypertrophie du scalène antérieur ${ }^{6,3}$; 38

2 L'hypertrophie du scalène moyen ${ }^{39}$

3 Une côte cervicale ${ }^{8}$

4 Une dérivation anormale du plexus-plus élevée ou plus basse que la normale ${ }^{6}$

5 Une chute anormale de l'épaule ${ }^{10}$

Aspects clınıques De façon caractéistıque, il apparaît une douleur à l'épaule et une senısbilité dans la région sus-clavicularre un ou plusieurs jours après l'opération

Tout le plexus peut être touché, en ce cas, le bras pend avec flaccidité et la peau de tout le bras est engourdie Seules les racines supérieures $(\mathbf{C} 5,6,7)$ peuvent être lésées, ce qui entraîne une rotation interne du bras, une extension de l'avant-bras et une pronation de la mann (paralysie de Erbs) Moins souvent, seules les racines inférieures (C8 et T1) peuvent être affectées, ce qui cause une perte de la flexion des doigts, une paralysie des muscles de la main et peut-être un syndrome de Horner (paralysie de Klumpke)

La lésion peut être lımitée à une seule racıne $S_{1}$ la racıne postérıeure est lésée, il y a perte de l'abduction du bras et paraly'se des extenseurs du coude, du porgnet et des doigts $S_{1}$ c'est la racine latérale qui est impliquée, ll y a paralysie des fléchisseurs du coude et du poignet S’ll s'agit de la racine médıale, la lésıon est semblable à celle qui affecte les racines inférieur es

Il peut être très difficile de déterminer exactement quelle région de la peau est engourdie, parce qu'il existe un croisement extensif de la distribution des nerfs cutanés Une méthode simple est de vérifier la partie posténeure du premier espace palmaire pour les lésions de la racine postérieure, la face palmaire de la troisième phalange de l'index pour la paralysie de la racine latérale, et la face palmarre de la troisième phalange de l'auriculaure pour l'anesthésie de la racine médiane

\section{B Le nerf radial}

Le nerf radial peut être blessé à l'endroit où 1 l traverse le bras sı on laısse pendre le bras à côté de la table d'opération $S_{1}$ le malade est en position horizontale, le plexus frotte contre le bord de la table $S_{1}$ le malade est en position de Trendelenburg, le bras a tendance à être poussé en haut contre le cerceau, tel que démontré dans la figure 6 , ll y a pincement du nerf entre le cerceau et la gouttière ${ }^{9}$

Cliniquement, il survient une chute du porgnet et l'extension de l'articulation 
métacarpo-phalangıenne est ımpossıble à cause de la paralysıe des muscles extenseurs de l'avant-bras Une farblesse de l'abduction du pouce est due à une paralusie du long abducteur. La surface dorsale des trois derniers dorgts et demi et la main adjacente présentent divers degrés d'engourdıssement, de sécheresse, de chaleur et de rougeur

Au point de vue médico-légal, cette blessure doit être dufférenciée d'une prémédication intramusculaire donnée plus bas que le deltoîde Une injection donnée à cet endroıt peut facilement léser le nerf radıal dans la gouttière en spirale

Un goutte à goutte.de thiopentone dans une veme passant au-dessus du côté latéral du poignet peut endommager le nerf radial superficiel, ${ }^{30}$ et causer une perte de sensibilité sur la face dorsale de l'éminence thénar

\section{Le nerf cubital}

Le nerf cubital peut aussi être comprimé par la tubérosité posténeure de l'épicondyle moyen de l'humérus ${ }^{41} \mathrm{La}$ compression peut être causée par le fer angle de la table, si on laisse le coude tomber légèrement sur le côté de la table Les blessures au nerf cubital ont été rapportées à la suite d'opérations où le bras du malade étant plé sur l'abdomen ou sur le thorax, ll s'en est survı une élongation du nerf autour de l'épicondyle médian de l'humérus par une flexion prononcée du coude, et la pression exercée par le poids du bras lur-même était apparemment suffisante pour produre une ischémıe Cela survient peut-être parce que dans plus de 20 pour cent des cas, le nerf cubital a un trajet plus médial que celur qui est décrit dans les volumes classiques Il passe derrière l'extrémité de l'épicondyle plutôt que dans le gouthère où il est pluś protégé (fig 7)

L'étreinte du côté cubital du poing est farble à cause de la paralysie du fléchisseur cubital du carpe et de la moitié interne du fléchisseur profond des doıgts La paralysie du fléchisseur profond des doigts affaiblit également la flexion des articulations interphalangiennes des deux doig's médians La flexion des artıculations métacarpo-phalangiennes et interphalangiennes proxımales, l'extension des articulations distales interphalangıennes, l'abduction et l'adduction des quatre doigts médians-lorsque le malade les étend-sont farbles à cause de la perte d'innervation des interosseux et du $3 \mathrm{e}$ et du 4e lombrics Il existe également une incapacité de farre l'abduction ou l'oppositıon de l'auricularre à cause de la paralysie des muscles hypothénar Il survient également une perte de sensibilité et d'orientation des deux surfaces du doıgt et demı mnervé par le médıan et de la surface adjacente de la main Eventuellement, les muscles intrinsèques de la main-à part l'éminence thénar-deviennent épuisés et des contractures s'installent entraînant la main caractérıstıque en forme de graffe

\section{Le nerf médian}

Le nerf médian est adjacent au cubital médian et anx veınes basiliques dans la fosse antécubitale, et 1 peut être lésé au cours d'une injection intraveineuse de thopentone, sort par le traumatisme direct de l'arguille, soit par l'extravasation du thiopentone De semblables lésions peuvent survenir même après une ponction veineuse et une fixation de l'anguille parfaitement réussie, s le malade bouge son bras à cause d'une anesthésie trop superficielle (fig 8) 
A l'examen du malade, il y a une mcapacité d'opposer le pouce à l'aunculaure (paralysie de l'opposant du pouce), faublesse de l'abduction du pouce (paralysie de l'abducteur du pouce) et perte de la flexion de la trossième phalange de l'index (paralysie de la moitié externe du fléchissieur profond des doigts) Eventuellement, l'éminence thénar s'aplatıt $\mathrm{La}$ sensibilite et la transpiration diminuent sur la face palmarre des trois doigts et demı et de la paume adjacente

\section{Le membre inférneur}

\section{A Nerf sciatique popluté externe}

Parmı les nerfs du membre inférieur, le nerf sciatıque poplité externe est le plus fréquemment lésé, cependant, 1 ne l'est pas aussi souvent que le plexus brachial Au cours de la position de lithotomie, ${ }^{43}$ ce nerf peut être comprimé contre la tête du péroné Dans cette position, la flexion des hanches et des genoux produit une élongation du nerf Le col du péroné frotte sur la tige de métal vertıcale à laquelle est attachée la courrose supportant le pied, ou sur un support métallique courbe soutenant le genou, ${ }^{1}$ aloıs, le nerf déjà trop tendu se trouve pincé (fig 9) Une blessure semblable peut également être provoquée par un bandage serré du genou ou par un tourniquet ${ }^{11}$

Les constatations physiques sont la chute du pied (paralysie du tibial anténeur, du long extenseur des orteils et du long extenseur du gros ortell) perte de l'extension dorsale des orteils (paralysie du long et du court extenseurs du gros orteil), incapacité d'éverser le pied (paralysie du long et du court péroniers) et engourdissement de la région latérale et antéro-latérale du mollet et de la moitié médiane de la face dorsale du pied

\section{$\mathrm{B}$ Le nerf saphène}

Le nerf saphène peut également être coincé contre le condyle médian du tibia si le pied est suspendu en étant appuyé latéralement à la tige verticale (fig 10) Il se développe une paresthésie le long du côté médian et antéro-médıan du mollet

\section{Le nerf scuatrque}

Chez un malade amaigrı, le nerf sciatıque peut être comprimé lorsqu'il sort du piriforme qui le couvrait et qu'il est appuyé sur une table dure, ceci se produit lorsqu'on élève la fesse du côté opposé au cours de l'enclouage d'une hanche ${ }^{12}$ Il peut être blessé également par une injection intramusculaure dans la fesse ${ }^{30}$

Il y a alors une paralysie de tous les muscles au-dessous du genou et peut-être aussı des muscles de la cuisse, aussı bien qu'un engourdıssement de la moitié externe du mollet et de la plus grande partie du pied, à l'ekception du bord interne de l'arche

\section{Le nerf honteux}

Le nerf honteux peut êtıe pressé contre la tubérosité de l'ischion à cause de la traction des deux jambes contre une tigge peu protégée, dans des cas d'orthopédie, Il s'ensuit une perte de la sensibilité di pérmé et une incontinence des selles ${ }^{44}$ 


\section{$\mathrm{E}$ Le nerf fémoral}

Le nerf témoral a déjà été lésé par une pression et une rotation latérale de la jambe par la branche d'un écarteur autostatıque employé au cours d'une laparotomie gynécologique ${ }^{45}$ (fig 11)

A l'examen, 1 y a une perte de flexion de la hanche et de l'extension du genou due à la paralysie du quadriceps crural La sensibilité et la fonction autonome sont disparus sur toute la région antérıeure de la cuisse, la régıon interne et antéro-1nterne du mollet

\section{F Le nerf obturateur}

Un cas de paralysie du nerf obturateur avec paralysie des adducteurs et engourdıssement du côté interne de la cuisse a été observée par les auteurs à la suite d'une anesthésie épıdurale La malade avart eu une délivrance difficile et avec application de forceps et l'obstétricien se rappelait qu'll avart été obligé de trer plus violemment sur le forceps qu'il ne l'avait jamais fart Alors, on croit que ce genre de blessure relève plutôt de l'obstétrique que de l'anesthésıe

\section{Le tronc}

\section{A Le nerf phrénqque}

On peut blesser le phrénique au cours de l'exécution d'un blocage du plexus brachial par la voıe sus-clavicularre, et 1 en résulte une paralysie et une élévation du diaphragme ${ }^{9}$

\section{B Le ganglion étoulé}

On peui léser le ganglion étollé, et 1 en résulte un syndrome de Claude Bernard Horner-myosis, enophtalmie, ptose de la paupière el sécheresse de la peau.

\section{Les nerfs spino-lombarres}

On peut blesser ces nerfs au cours d'une tentative de blocage sympathique lombaire, il en résulte des douleurs dans le territorre où se distribuent les nerfs touchés

\section{La tête}

\section{A Le nerf optrque}

Des lésions à ce nerf ont de très sérneuses conséquences pour le malade et elles sont, malheureusement, assez fréquentes La pression exercée sur le globe oculaure, partıculıèrement au cours de l'hypotension contrôlée ou accidentelle, peut causer une thrombose de l'artère rétınienne centrale avec perte de la vue au réveıl de l'anesthésie Cette lésion constitue une exception à la règle d'après laquelle lischémie est d'ongine intraneurale De plus, 1 peut y avoir une désorganisation complète du globe ocularre

Une telle pression est exposée à survenir en position ventrale, et c'est exactement dans cette position qu'on emploie l'hypotension contrôlée, par exemple, au cours de la greffe lombarre ou de l'insertion de la tige Harrington Walkup 
rapporte deux cas de cécité unilatérale à la sute d'une pression éxercée sur le globe oculaure par un support en forme de fer à cheval, au cours d'une résection pulmonaure pratiquée en position ventrale, ${ }^{48}$ les auteurs ont eu connassance d'un cas semblable qui s'est produit avec la même sorte d'appure-tête au cours d'une opération en neurochirurgie Une compression semblable de l'oell peut également survenir sı un appure-tête Barley glısse sur l'oèl au cours de la pratıque d'un volett osseux de la fosse posténeure en position assise ${ }^{1}$ Le débit sanguin dans l'artère rétınienne centrale est vraisemblablement dımınué à cause de la position assise (fig 12) Une lésion semblable à l'oeil peut être produite par la pression d'un masque facial qui est trop grand pour le malade

A l'examen, au cours des 24 premières heures, la pupille demeure dilatée et elle réagit consensuellement mais non pas directement à la lumière La cornée est légèrement ombragée et les paupières|sont souvent oedémateuses Les arténoles de la rétıne sont dılatées et les veines, engorgées Il existe de loedème de la macule et de la rétıne aux environs du disque opt- pue $S_{1}$ la blessure est grave, Il peut $\mathrm{y}$ avoir un point rouge cerse dans la macule Au bout de quelques jours, le disque optıque devient blanc perlé Les artérıoles sont teut étroites comme des fils blancs La macule et la rétıne pérıpapillaire deviennent pıgmentées de façon diffuse et 1 apparaît comme une sorte d'escarre sur la macule ${ }^{47}$

\section{B Le nerf sus-orbitarre}

On peut comprimer ce nerf avec le connecteur métallique d'un tube endotrachéal sı le matelassage n'est pas adéquat (fig 13) Il apparaît de la photophobie, de l'engourdissement du front et de la douleur dans l'oell ${ }^{48}$

\section{Le nerf facial}

Il est possible de pincer le nerf facial entre les doigts et la branche montante du maxillaure si l'on exerce une pression exagérée en avant pour maintenır les voies respiratoires libres (fig 14)

Il survient une chute de la commissure des lèvres, la salive coule, la mastication est difficile et le côté malade de la figure est plus doux que la normale ${ }^{49}$

\section{La branche buccale du nerf facial}

On a rapporté des lésions à cette branche nerveuse parce que la pression exercée par les courroies ou par le masque était trop grande Cette blessure est plus susceptible de survenir si le trajet du nerf est superficiel par rapport à la glande parotide Il y a perte de fonction du muscle orbicularre de la bouche ${ }^{30}$ (fig 15)

\section{E Nerf moteur oculare externe}

Dans les revues médıcales, on parle de la paralysie de tous les nerfs crânıens excepté du 10e, à la surte de rachianesthésie mais, dans 90 pour cent des cas, c'est le 6e nerf qui est touché La fréquence est probablement plus grande que ne le font croire les rapports, en effet, plusieurs de ces cas peuvent passer maperçus, car le malade ne se plaint que d'un léger trouble de la vue qui disaparaît dans quelques jours Dans la majorité des cas rapportés, on a observé de la diplopie 
précédée de violents maux de tête, de raıdeur de la nuque, de nausées, d'étour $\nmid$ dissements et de photophobie Linstallation de la paralysie peut se faure au cours des 21 premiers jours après l'opération ${ }^{30}$ et la guérison n'être complète que deux. ans après l’opération ${ }^{50}$

\section{F Le nerf triumeau}

L’analgésie du trujumeau est la plus fréquente manifestation toxıque des produits de dégradation du trichloréthylène Toutefors, on a décrit des lésions intéressant tous les nerfs crânıens excepte le 8e

De l'engourdissement et une sensation de froid autour des lèvres commencent un ou deux jours après l'administration de l'agent anesthésıque Au cours des quelques jours qui suivent, la zone d'analgésie ou de perte de sensibilité s'étend pour couvrir tout le territorre innervé par le trijumeau. On peut observer de la dufficulté à mastıquer, et même une chute du maxillaure De plus, il peut apparaître des sıgnes physiques laıssant supposer que d'autres nerfs crânıens com. mencent à paralyser Le taux de mortalité est assez élevé à cause d'un type d'encéphalıte Toutefors, sı le malade survit, ${ }^{28}{ }^{29}$ la guélison complète se produit dans environ huit semames

\section{Trattement}

Sans aucun doute, c'est la prévention qui demeure le tratement de choix ${ }^{651}$ Il faut éviter l'usage des supports à bras, spécialement au cours de la position de Trendelenburg Il est préférable de placer les bras le long du corps du malade, de les envelopper d'un champ ou d'un protecteur en métal Si l'on emploie ce protecteur en métal, il faut matelasser bien soigneusement la partie intérieure de ce protecteur $S_{1}$ l'on se sert d'un champ pour envelopper le bras, ll faut avertir les chirurgiens, partıculıèrement les très obèses, de ne pas se servir du bras du malade comme support pour leur abdomen S'il faut absolîment pratıquer l'ab duction du bras, le support ne doit pas faure un angle de plus de $60^{\circ}$ avec la table d'opération Le bras doit être placé en pronation

Les épauhières doivent être bien matelassées et placées vis-à-vis de l'acromın et non pas sur les clavicules On peut aussi employer des supports spéciaux fixés au pied de la table pour empêcher que le malade ne glisse vers le bas ${ }^{11}$ Un matelas spécial qui ne glısse pas peut également être employé pour arriver à la même fin ${ }^{5253}$ De toute façon, Il ne faut jamass suspendre le malade par les poignets Il faut renoncer à l'usage de la position de Trendelenburg trop pro noncée ${ }^{54}$ et de volumineux supports pour le fore

Lorsqu'on met un malade en position de lithotomie, on doit placer un coussin entre les jambes et la tige de métal verticale ou entre les genoux et le support Les supports orthopédıques doivent également être bien matelassés

Dans la région de la tête, il faut également se servir de coussinets entre le connecteur du tube endotrachéal et la figuse du malade Les courrores pour les masques ne doivent pas être trop serrées Il faut éviter une piession excessive poussant le maxillare en avant ainsı que les positions anormales de la tête Il ne faut pas exercer de pression sur les yeux, et il est prudent de signaler ce fait su le dossier 
En pratiquant des anesthésies régıonales ou des anesthésies locales, il faut employer des argulles de fin calıbre dont les lèvies du biseau sont arrondies ${ }^{55}$; ces aigulles pourront s'appuyer sur les fibres de la dure-mère sans les couper. ${ }^{.6}$ Il faut éviter les tentatives multıples pour faure l'injection et s'assurer d'une stérilité rigoureuse au cours de la technique

Il est absolûment interdit d'employer le curcunt fermé avec absorptıon de $\mathrm{CO}_{2}$ lorsque l'on administre du trichloréthylène ou du chloroforme.

Il ne faut pas pratıquer une anesthésie locale $n \mathfrak{n}$ une injection intraveineuse sur un membre déjà atteint d'une lésion nerveuse

Lorsque survient une paralysie nerveuse, il peut' être nécessaure de recourrr à l'administration d'analgésiques au cours des premiers jours pour contrôler la douleur La peau dépourvue de sensibilité doit être protégée contre les blessures et contre l'ulcération La mobılité artıculaure doit être conservée par la pratıque de massages et de manipulations Les contractures muscularres peuvent être prévenues en immobilisant le membre Il s'impose de pratıquer tous les jours une stımulation galvanıque intermittente des muscles atteints L'exercice, d'abord passif puis ensuite actıf, doit être commencé aussitôt que possible

S'll n'y a pas de sıgne de guérison après, un essal consciencieux d'un trattement conservateur, et s'il n'y a pas de réponse à la stımulation galvanıque appliquée près du site de la lésion, il faut songer à la chirurgie Cette chirurgie peut consister en l'excision des tissus cicatriciels pour faciliter la reconstitution du nerf, ou en la transplantation de muscle ou de tendons pour compenser l'incapacité fonctionnelle

Lorsqu'll survient une paralysie du 6e nerf, l'œil affecté devrait être fermé pour éviter la diplopie et prévenir les nausées. Les exercices musculaires des yeux et la rééducation combinée peuvent être utiles $\mathrm{S}_{1}$, après deux ans, la guérison n'est pas obtenue, 1 peut devenir nécessaure de recourir à la chirurgie.

\section{RÉSUMÉ}

Nous avons passé en revue les différentes sortes de blessures nerveuses rencontrées au cours de l'anesthésie Nous en avons souligné les facteurs éliologiques, la symptomatologie, la prévention et le traitement 